\title{
Postprocedural CT for perivertebral cement leakage in percutaneous vertebroplasty is not necessary-results from VERTOS II
}

\author{
Alexander Venmans • Caroline A. Klazen • \\ Willem Jan van Rooij • Jolanda de Vries • \\ Willem P. Mali • Paul N. Lohle
}

Received: 8 March 2010/Accepted: 12 April 2010/Published online: 5 May 2010

(C) The Author(s) 2010. This article is published with open access at Springerlink.com

\begin{abstract}
Introduction During percutaneous vertebroplasty (PV), perivertebral cement leakage frequently occurs. There is some concern that cement deposits may migrate towards the lungs via the veins during follow-up. We used baseline and follow-up computed tomography (CT) to assess the incidence and extend of late cement migration in a large consecutive patient cohort.

Methods VERTOS II is a prospective multicenter randomized controlled trial comparing PV with conservative therapy for osteoporotic vertebral compression fractures (OVCFs). Patients assigned to PV had baseline postprocedural CT scans of the treated vertebral bodies. After a mean follow-up of 22 months, 54 of 78 patients (69\%) had follow-up CT. CT scans were analyzed and compared for perivertebral venous, discal, and soft tissue leakage.

Results Perivertebral cement leakage occurred in 64 of 80 treated vertebrae $(80 \%$; $95 \% \mathrm{CI}, 70 \%$ to $87 \%)$. All patients remained asymptomatic. Perivertebral venous leakage was present in 56 vertebrae (88\%), mostly in the anterior external venous plexus (46 of $56,82 \%$ ). Discal leakage occurred in 22 of 64 vertebrae (34\%) and soft tissue
\end{abstract}

A. Venmans $(\bowtie) \cdot$ C. A. Klazen $\cdot$ W. J. van Rooij $\cdot$ P. N. Lohle Department of Radiology, St. Elisabeth Ziekenhuis Tilburg,

Hilvarenbeekseweg 60,

5022 GC Tilburg, The Netherlands

e-mail: alexandervenmans@hotmail.com

J. de Vries

Department of Medical Psychology, St.

Elisabeth Ziekenhuis Tilburg,

Tilburg, The Netherlands

W. P. Mali

Department of Radiology, University Medical Centre Utrecht, Utrecht, The Netherlands leakage in two of 64 (4\%). Mean injected cement volume in vertebrae with leakage was higher $\left(4.5\right.$ versus $3.7 \mathrm{~cm}^{3}$, $p=0.04$ ). Follow-up CT scan showed unchanged perivertebral cement leakages without late cement migration.

Conclusion Perivertebral cement leaks during PV for OVCFs occurred frequently in the VERTOS II trial. Cement leakage occurred more frequently with higher injected volumes. However, all patients remained asymptomatic, and late cement migration during follow-up did not occur. Standard postprocedural CT of the treated vertebral body in PV is not necessary.

Keywords Percutaneous vertebroplasty · Cement leakage CT scan

\section{Introduction}

Perivertebral leakage of cement during percutaneous vertebroplasty (PV) has been reported to occur frequently in up to $65 \%$ of treated osteoporotic vertebral compression fractures (OVCFs) [1, 2]. Most of these leakages cause no clinical symptoms, but pulmonary embolism and neurological complications have occasionally been reported [3, 4].

VERTOS II is a randomized controlled trial comparing PV and conservative therapy for OVCFs in 202 patients. In this trial, patients assigned to PV had a standard postprocedural computed tomography (CT) scan of the treated OVCF with the aim to assess the patterns of perivertebral cement leakage and its possible clinical impact. There is some concern that cement deposits may migrate to the lungs via the veins during follow-up; sharp and elongated spike-like cement fragments might cause perforation of vessels or heart. In addition, local damage to the adjacent anatomical 
structures by the leaked cement may cause symptoms like soft tissue hematoma or radiculopathy [5-8]. In this study, we used baseline and follow-up CT to assess the incidence, anatomical location, and clinical impact of perivertebral cement leakage on short- and long-term in a large patient cohort.

\section{Methods}

Patients

The study protocol of the VERTOS II trial has been described in detail elsewhere [9]. In short, VERTOS II was an unmasked randomized controlled trial in five large teaching hospitals in the Netherlands and one in Belgium. The protocols of VERTOS II and the present study were approved by the institutional review board at each participating centre. Between October 2005 and June 2008, 202 patients were randomized for PV and conservative therapy. All patients assigned to PV had baseline postprocedural CT scans of the treated vertebral bodies. Ultimately, in 98 patients, PV was performed without clinical procedural related complications. These 98 patients form the bases of the present study. During a mean followup of 22 months (median, 21 months; range, 6-42 months), ten patients died, and six refused to complete the protocol of VERTOS II. The remaining 82 patients were invited by telephone for a native CT scan of the treated vertebra to detect possible migration of the perivertebral cement leakages and evaluation of possible local pathology related to the cement leakage.

Twenty-four patients declined participation, and four patients could not be reached. Thus, 54 of 82 patients (69\%) had follow-up CT. There were 36 women $(67 \%)$ and 18 men (33\%) with a mean age of 74 years (median, 77; range, 53-88 years). These 54 patients had 80 OVCFs and were treated in 60 sessions. Thirty-nine patients were treated for one OVCF, 11 patients for two, and four patients for three OVCFs in one session. During follow-up, four patients presented with a new OVCF and were treated again. One of these four patients had two additional PVs, for one OVCF each time. Location of treated vertebra is displayed in Fig. 1.

\section{PV technique}

PV was performed on a single or biplane angiographic unit under fluoroscopic guidance. After local infiltration analgesia (Lidocain 1\%, Braun, Melsungen, Germany), needles were bilaterally transpedicular inserted into the vertebral body. Polymethylmethacrylate bone cement (Osteo-firm ${ }^{\circledR}$, COOK Medical, Bloomington, IN, USA) was injected

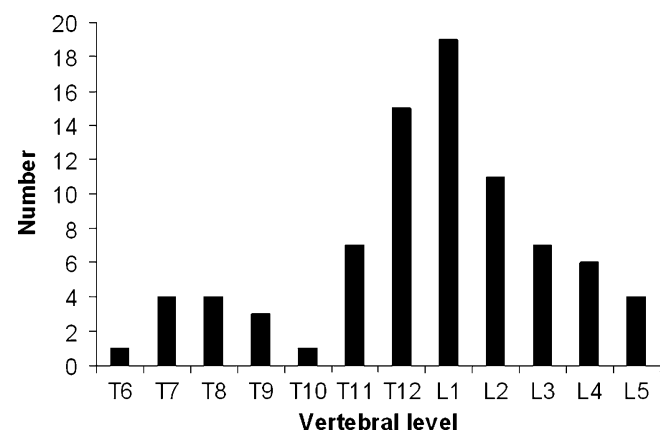

Fig. 1 Distribution of 54 treated osteoporotic compression fractures

under continuous lateral fluoroscopy alternating both pedicles using $1-\mathrm{cm}^{3}$ syringes. Injection was stopped whenever perivertebral cement migration was observed. Injection was resumed after a 15-20-s delay without changing needle position. Volume of injected cement in each treated vertebral body was recorded. Immediately after the procedure, a CT scan of the treated OVCF was performed to evaluate perivertebral cement leakage.

Perivertebral cement leakage on postprocedural and follow-up CT scan

Treated vertebrae were assigned into three location categories: T5-T10, T11-L2, and L3-L5. The anatomical location of perivertebral venous cement leakage was recorded according to the plexus venosus vertebralis, a venous network that extends along the entire length of the vertebral column (Fig. 2). In addition to the venous location, discal and soft tissue leakages were recorded.

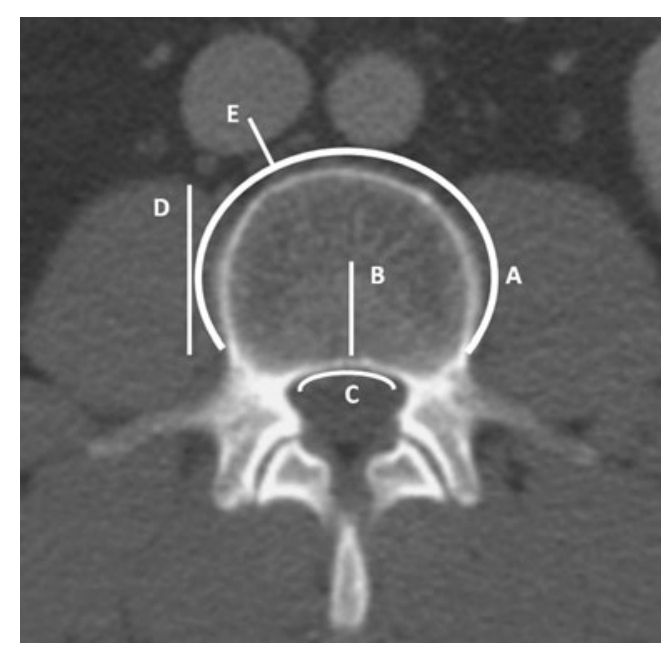

Fig. 2 Schematic representation of patterns of perivertebral venous cement leakage. Leakage into the anterior external venous plexus $(A)$, basivertebral vein $(B)$, anterior internal venous plexus $(C)$, segmental vein $(D)$, inferior caval vein, or (hemi)azygos vein $(E)$ 
Data analysis

Chi-square test was used to correlate perivertebral cement leakage with the location of treated OVCFs into three categories. Mean volume of injected cement in vertebrae with leakage was compared to mean volume of cement in vertebrae without leakage using the $t$ test. Statistics were performed with SPSS version 15.0.1. The VERTOS II study is registered with ClinicalTrials.gov, with the number NCT00232466.

Role of the funding source

The sponsors of the study had no role in study design, data collection, data analysis, data interpretation, writing of the report, or the decision to submit the paper for publication.

\section{Results}

Perivertebral cement leakage on postprocedural and follow-up CT scan

Any perivertebral cement leakage was observed in 64 of 80 treated vertebrae $(80 \% ; 95 \% \mathrm{CI}, 70 \%$ to $87 \%)$. Discal leakage was present in 22 vertebrae (34\%), in eight vertebrae (13\%) in combination with venous leakage. Perivertebral soft tissue leakage occurred in two vertebrae (4\%). Altogether, 56 of 64 vertebrae $(88 \%)$ had cement leakage into the perivertebral venous system. Cement in the anterior external venous plexus was observed in 46 of 56 vertebrae (82\%), in 32 vertebrae $(57 \%)$ in combination with cement in a segmental vein. Five vertebrae $(9 \%)$ had cement in the inferior caval vein and six $(11 \%)$ in the azygos vein, all in combination with cement in a segmental vein and the anterior external venous plexus. Cement in the basivertebral vein was present in 30 of 56 vertebrae (54\%), in the anterior internal venous plexus in 33 $(59 \%)$ and both in the basivertebral vein and anterior internal venous plexus in 26 (46\%). Three vertebrae (5\%) had cement in the intervertebral vein, all in combination with cement in the anterior external venous plexus, basivertebral vein, and anterior internal venous plexus. No cement leaks were seen in the posterior internal and external venous plexus.

Comparison of follow-up CT scan (mean, 22 months; median, 21 months; range, 6-42 months) of treated vertebrae with baseline CT showed unchanged anatomical location of the perivertebral cement leakages in all vertebrae without late cement migration.

\section{Data analysis}

Chi-square test showed no statistical relation between location of the treated vertebra and the occurrence of perivertebral cement leakage $(p=0.64)$.
Mean volume of injected cement in 47 vertebrae with leakage was $4.5 \pm 1.8 \mathrm{~cm}^{3}$, and in 33 vertebrae without leakage, this was $3.7 \pm 1.6 \mathrm{~cm}^{3}$. This difference was significant ( $p=0.04 ; 95 \% \mathrm{CI},-1.58 \%$ to $-0.02 \%$ ).

\section{Discussion}

In this well-defined and large patient cohort from VERTOS II, we found that during PV, perivertebral cement leakage occurred in more than half of the treated vertebrae. Most leakages were in perivertebral venous structures, leakage into the disk or perivertebral soft tissues was infrequent. Cement leakage occurred more frequent with higher volumes of injected cement. Follow-up CT after almost 2 years showed that late migration of leaked cement deposits did not occur. Clinically, patients remained asymptomatic; there were no symptomatic pulmonary emboli, and radiculopathy or soft tissue hematoma did not occur. Our findings suggest that standard postprocedural CT scan after PV is not warranted and should be confined to clinically symptomatic patients only. Omitting CT from the PV protocol is cost-effective and reduces radiation burden.

Knowledge of the anatomy of the perivertebral veins is helpful in understanding the venous leakage patterns on CT. Leakage more often occurs in the perivertebral venous plexus than in adjacent disks or perivertebral soft tissues. The venous complex along the vertebral column consists of three major intercommunicating networks $[10,11]$ : the internal and the external venous plexus and the basivertebral system. The basivertebral system is oriented horizontally in the centre of the upper half of the vertebral body. The basivertebral veins originate in the ventral third of the vertebral body and converge posteriorly to drain into the ventral part of the internal venous plexus, sometimes as a single vein and sometimes as two separate tributaries. Anteriorly, the basivertebral veins join the external plexus. The exiting point of the basivertebral vein on the dorsal surface of the vertebral body is located in the middle between the pedicles. The anterior internal venous plexus drains into the segmental veins that exit the spinal canal through the foramen, between the nerve root and the medial wall of the pedicles. This means that there is a direct venous connection between the bone marrow and the foraminal space.

Comparison of frequency of cement leakage between studies is hampered by differences in methods used. Detection rates in studies using intraoperative fluoroscopy only instead of $\mathrm{CT}$ will be substantially lower since sensitivity of CT is much higher. In two studies about frequency of local cement leakage that used postprocedural CT for detection, rates of $63 \%$ and $81 \%$ were found, comparable to our $80 \%[12,13]$. 
Cement leakage during PV seems to be largely inevitable according to the high reported rates in this study and in the literature. Small leakages are without clinical consequences. With proper use of technique and fluoroscopy, clinical relevant cement leakage should be avoided.

\section{Conclusion}

Perivertebral cement leaks during PV for OVCFs occurred frequently in the VERTOS II trial. Cement leakage occurred more frequently with higher injected volumes. However, all patients remained asymptomatic, and late cement migration during follow-up did not occur. Standard postprocedural CT of the treated vertebral body in PV is not necessary.

Conflict of interest statement We declare that we have no conflict of interest.

Open Access This article is distributed under the terms of the Creative Commons Attribution Noncommercial License which permits any noncommercial use, distribution, and reproduction in any medium, provided the original author(s) and source are credited.

\section{References}

1. Jensen M, Evans A, Mathis J (1997) Percutaneous polymethylmethacrylate vertebroplasty in the treatment of osteoporotic vertebral body compression fractures: technical aspects. AJNR Am J Neuroradiol 18:1897-1904

2. Cortet B, Cotton A, Boutry N (1999) Percutaneous vertebroplasty in the treatment of osteoporotic vertebral compression fractures: an open prospective study. J Rheumatol 26:2222-2228
3. Venmans A, Lohle P, van Rooij W et al (2008) Frequency and outcome of pulmonary polymethylmethacrylate embolism during percutaneous vertebroplasty. AJNR Am J Neuroradiol 29:1983-1985

4. Nussbaum D, Gailloud P, Murphy K (2004) A review of complications associated with vertebroplasty and kyphoplasty as reported to the food and drug administration medical device related web site. J Vasc Interv Radiol 15:1185-1192

5. Mathis J (2002) Procedural techniques and materials: complication avoidance and tricks of the trade. AJNR Am J Neuroradiol $119-120$

6. Mathis J (2003) Percutaneous vertebroplasty: complication avoidance and technique optimization. AJNR Am J Neuroradiol 24:1687-1706

7. Hodler J, Peck D, Gilula L (2003) Midterm outcome after vertebroplasty: predictive value of technical and patient-related factors. Radiology 227:662-668

8. Diamond T, Bryant C, Browne L (2006) Clinical outcomes after acute osteoporotic vertebral fractures: a 2-year non-randomised trial comparing percutaneous vertebroplasty with conservative therapy. Med J Aust 184:113-117

9. Klazen C, Verhaar H, Lampmann L (2007) Percutaneous vertebroplasty versus conservative therapy in patients with painful osteoporotic vertebral compression fractures; rationale, objectives and design of a multicenter randomized controlled trial. Trials $8: 33$

10. Groen R, du Toit D, Philips F (2004) Anatomical and pathological considerations in percutaneous vertebroplasty and kuphoplasty: a reappraisal of the vertebral venous system. Spine 29:14651471

11. Kasó G, Horváth Z, Szenohradszky K (2008) Comparison of CT characteristics of extravertebral cement leakages after vertebroplasty performed by different navigation and injection techniques. Acta Neurochir 150:677-683

12. Yeom J, Kim W, Choy W (2003) Leakage of cement in percutaneous transpedicular vertebroplasty for painful osteoporotic compression fractures. J Bone Joint Surg Br 85:83-89

13. Schmidt R, Cakir B, Mattes T (2005) Cement leakage during vertebroplasty: an underestimated problem? Eur Spine J $14: 466-473$ 